\title{
Placental Growth, Fetal Growth and Maternal RhE Genotype
}

\author{
Egidio Bottini and Fulvia Gloria-Bottini \\ Chair of Preventive and Social Pediatrics, \\ University of Rome Tor Vergata, School of \\ Medicine, Rome, Italy
}

The Rh locus is composed of two genes RhD, which encodes the major $\mathrm{D}$ antigen and is present only in Rh-positive genomes, and RhCE, which encodes both the $\mathrm{Cc}$ and Ee polypeptides, most likely by alternative splicing events. Several membrane components, including $\mathrm{Rh}$ proteins and other glycoproteins recently characterized, are probably different subunits of an oligomeric complex with transport functions in the erythrocyte [3].

In previous papers we have reported an association of maternal $\mathrm{RhE}$ phenotype with neonatal macrosomia [1] and with metabolic control [2] in diabetic pregnant women. The recent discoveries on the structure and functions of $\mathrm{Rh}$ proteins prompted us to look for possible association of RhE phenotype with placental weight and birth weight in normal pregnancy.

260 Consecutive puerperae along with their newborn babies have been examined in the population of Penne. 295 Diabetic puerperae (including gestational and pre-existing IDDM and NIDDM) from the population of Rome along with their newborn babies have also been considered. Some data on these diabetic women have been reported in previous studies [1,2]. We have now considered also placental weight not examined in our previous reports.

Table 1 shows the relationship of maternal $\mathrm{RhE}$ phenotype with birth weight and placental weight. In healthy puerperae there is a strong

Table 1

Birth weight and placental weight in relation to RhE maternal phenotype in healthy puerperae from Penne

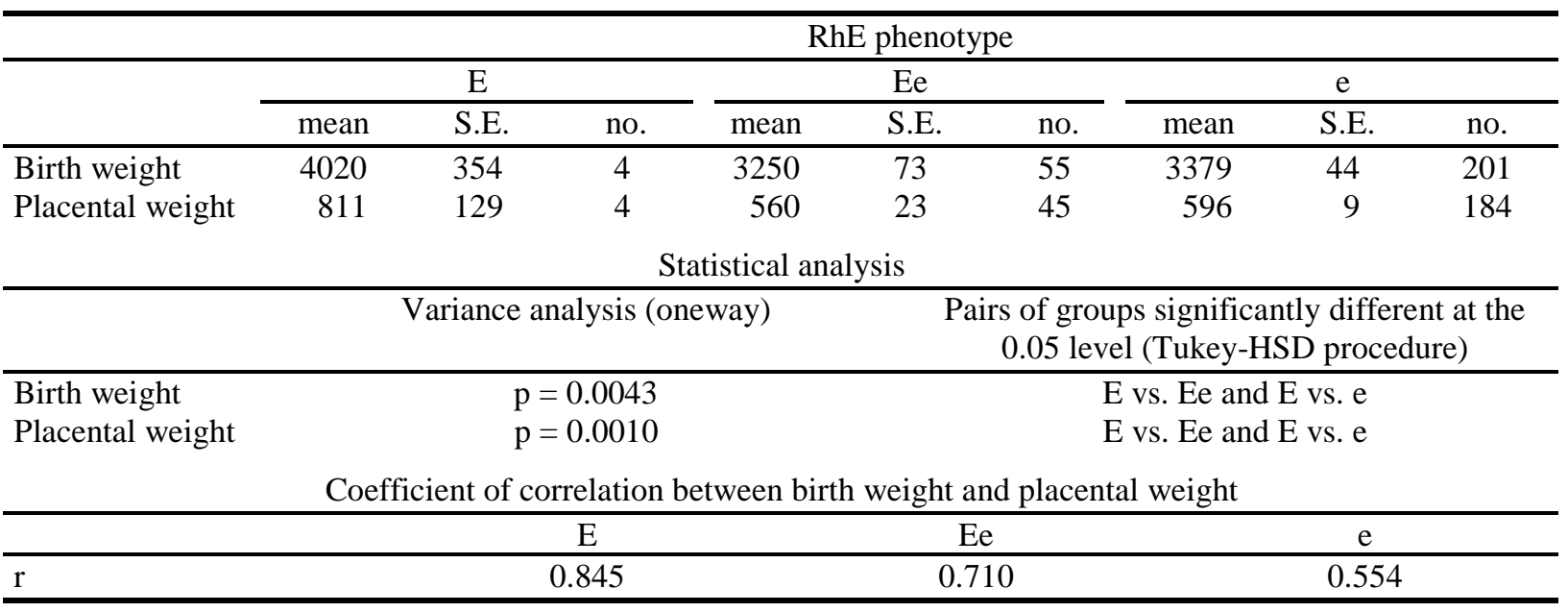


Table 2

Birth weight and placental weight in relation to RhE maternal phenotype in diabetic puerperae from Rome

\begin{tabular}{|c|c|c|c|c|c|c|c|c|c|c|}
\hline & \multicolumn{10}{|c|}{ RhE phenotype } \\
\hline & \multicolumn{3}{|c|}{$\mathrm{E}$} & \multicolumn{3}{|c|}{$\mathrm{Ee}$} & \multicolumn{3}{|c|}{$\mathrm{e}$} & \multirow{2}{*}{$\begin{array}{c}\text { Variance analysis } \\
\text { (p) }\end{array}$} \\
\hline & mean & S.E. & no. & mean & S.E. & no. & mean & S.E. & no. & \\
\hline Birth weight & 3605 & 388 & 4 & 3391 & 133 & 64 & 3417 & 51 & 227 & N.S. \\
\hline Placental weight & 900 & 70 & 4 & 688 & 24 & 64 & 697 & 14 & 227 & 0.079 \\
\hline \multicolumn{11}{|c|}{ Difference of placental weight between $\mathrm{E}$ and other phenotypes (two tail p) } \\
\hline \multicolumn{7}{|c|}{ E vs. Ee } & \multicolumn{3}{|c|}{$\mathrm{p}=0.023$} & \\
\hline & \multicolumn{3}{|c|}{ E vs. e } & & & & \multicolumn{3}{|c|}{$\mathrm{p}=0.052$} & \\
\hline
\end{tabular}

association of $\mathrm{RhE}$ with both developmental parameters showing the highest values of birth weight and placental weight in E mothers and the lowest values in Ee mothers. The pattern of relationship is similar in diabetic pregnancy but less marked (Table 2). Separate analysis of the three types of diabetes has not shown differences in the pattern of relationship between placental weight and $\mathrm{RhE}$.

It is interesting to note that correlation between birth weight and placental weight increases with the dose of *E "allele", showing the highest value in $* \mathrm{E} / * \mathrm{E}$ and the lowest in $* \mathrm{e} / *^{\mathrm{e}}$.

Overall the effect of $\mathrm{RhE}$ seems somewhat more marked on placental weight than on birth weight. The values of eta squared (a measure of the strength of association) are the following: RhE-placental weight association 0.06 and 0.02 for normal and diabetic pregnancy respectively, RhE-birth weight association 0.04 and 0.001 .

The similarity of the pattern in different population and in presence of diabetes - a situation associated with severe disturbances of intra-uterine growth - argues against a mere sampling artifact.
At present it cannot be excluded that the association with developmental parameters is due to some gene near to $\mathrm{Rh}$ and in linkage disequilibrium with it. The new acquisitions on the structure and the possible function of $\mathrm{Rh}$ proteins may suggest a causal involvement of $\mathrm{Rh}$ proteins on intrauterine development through its transport function.

\section{References}

[1] Gloria-Bottini, F., Gerlini, G., Lucarini, N., Amante, A., Lucarelli, P., Borgiani, P. and Bottini, E. Both maternal and foetal genetic factors contribute to macrosomia of diabetic pregnancy. Human Heredity 44, (1994) 24-30.

[2] Gloria-Bottini, F., Lucarini, N., Gerlini, G., Fallucca, F. and Bottini, E. Rh-PGM1 haplotype and clinical severity of IDDM during pregnancy. Diabetes Res. 13, (1990) 49-50.

[3] Telen, M.J. Erythrocyte blood group antigens: not so simple after all. Blood 85, (1995) 299306. 


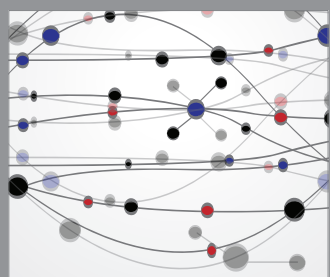

The Scientific World Journal
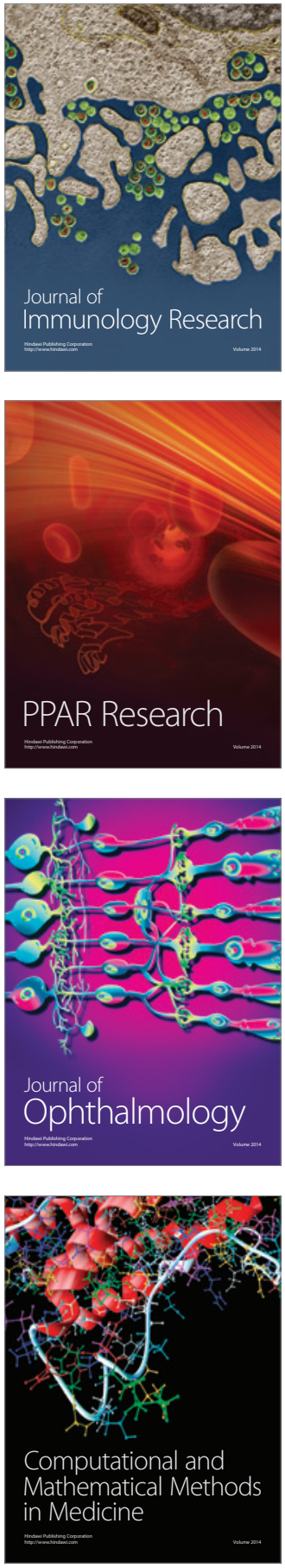

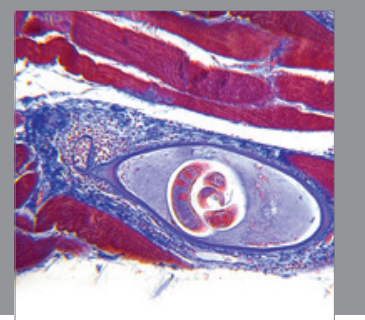

Gastroenterology

Research and Practice
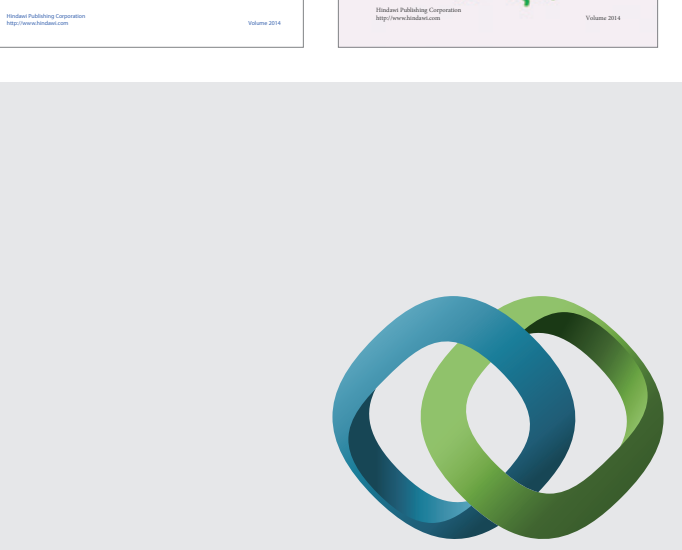

\section{Hindawi}

Submit your manuscripts at

http://www.hindawi.com
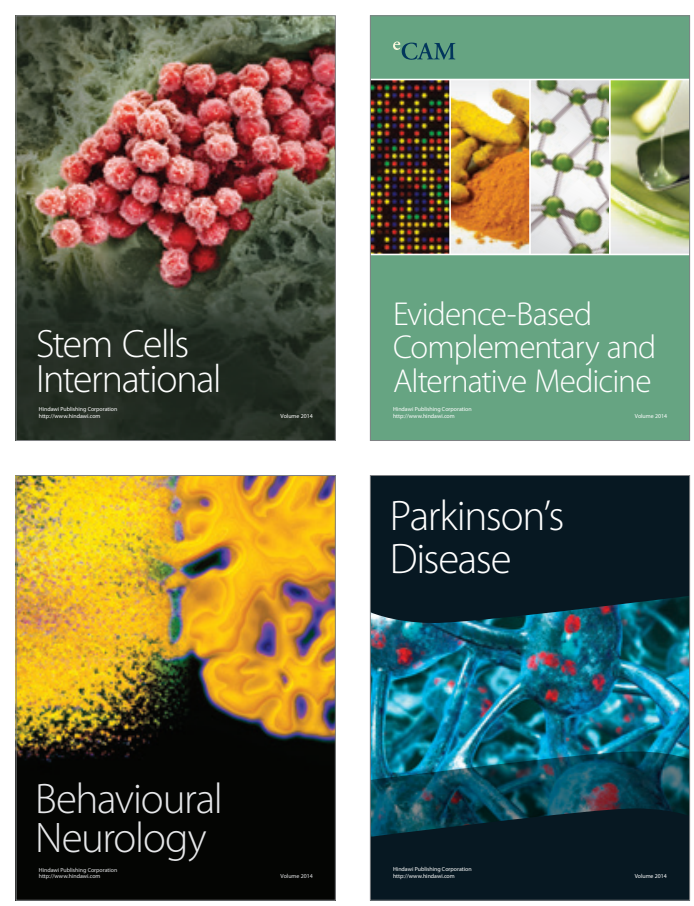

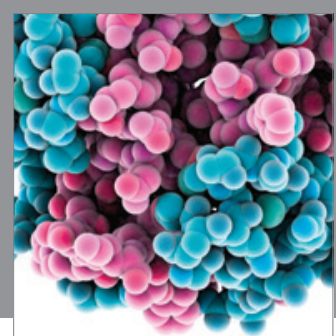

Journal of
Diabetes Research

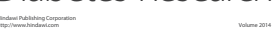

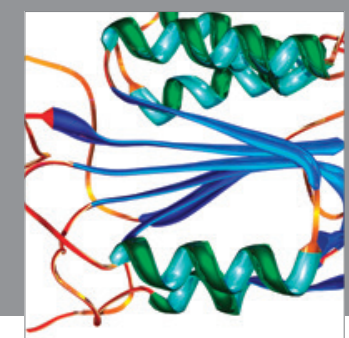

Disease Markers
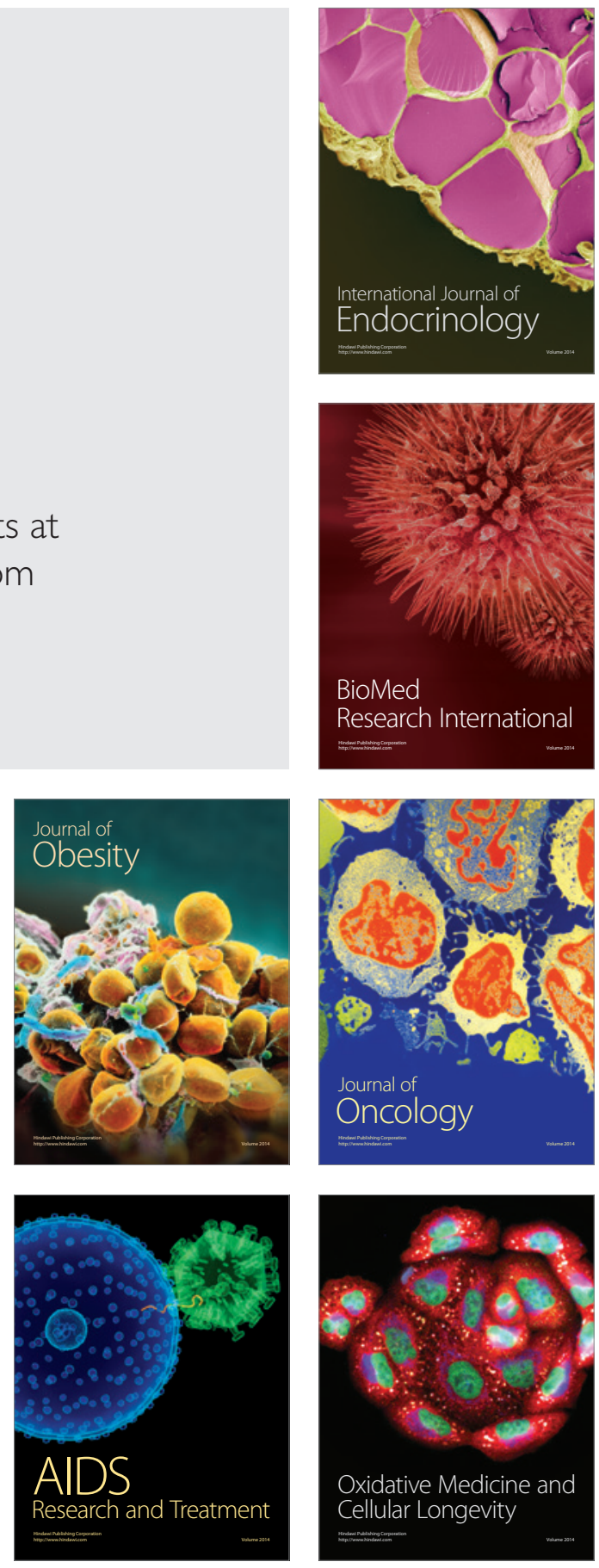